\title{
Participation in a workplace smoking cessation program incentivized by lowering the cost of health care coverage: Findings from the LLUH BREATHE cohort
}

\author{
Olivia Moses',2, Brenda Rea², Ernie Medina², Dennys Estevez², Josileide Gaio², Mark Hubbard', Kelly Morton ${ }^{3}$, Pramil N. Singh ${ }^{2,4}$
}

\begin{abstract}
INTRODUCTION Systematic analyses of workplace smoking cessation programs indicate that efficacy can be enhanced by using incentives. There is variation in the type of incentives used and their effect on participation and efficacy. The aim of our study was to examine whether lowering employee health plan costs (employee contributions, co-pays) encourage employee smokers to participate in workplace smoking cessation.

METHODS We conducted a 2014-2015 prospective cohort study of 415 employee smokers of Loma Linda University Health (LLUH). The employees were offered participation in a workplace smoking cessation program (LLUH BREATHE Initiative) with the incentive of enrollment in an employer-provided health plan that had a $50 \%$ lower employee monthly contribution and co-payment relative to the employer-provided health plan for non-participants. Participation rates and variables associated with participation were analyzed.

RESULTS In the LLUH BREATHE cohort, we found a very high rate of participation (72.7\%; 95\% CI: 69-77\%) in workplace smoking cessation that was encouraged by a lower out-of-pocket health plan cost for the participating employee and/or spouse. Participation did, however, vary by gender and spouse, whereby female employee households with a qualifying smoker were more than two times more likely (employee: $\mathrm{OR}=2.89,95 \% \mathrm{CI}$ : $1.59-5.24$; or spouse: OR $=2.71,95 \% \mathrm{CI}$ : $1.47-5.00)$ to participate in smoking cessation than male employee households. The point prevalence, at four months, of abstinence from smoking among the participants was 48\% (95\% CI: 42-54\%).

CONCLUSIONS Our findings indicate that a workplace smoking cessation program that uses a novel reward-based incentive of lower out-of-pocket health plan costs results in a participation rate that is much higher than US norms.
\end{abstract}

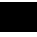

AFFILIATION

1 Risk Management, Loma Linda University, Loma Linda, United States

2 School of Public Health, Loma Linda University, Loma Linda,

United States

3 School of Behavioral Health,

Loma Linda University, Loma Linda, United States

4 Transdisciplinary Tobacco Research Program, Cancer Center, Loma Linda University, Loma Linda, United States
\end{abstract}

CORRESPONDENCE TO

Pramil N. Singh. School of Public

Health, Loma Linda University, Loma Linda, CA 92350, United

States. E-mail: psingh@llu.edu

KEYWORDS

smoking, health services, employee health

Received: 30 October 2019

Revised: 6 February 2020

Accepted: 17 February 2020

\section{INTRODUCTION}

One of the Healthy People 2020 goals for adult smoking cessation is to increase the rate of quit attempts in adult smokers to $80 \%$ or more; current national data sources (i.e. National Health Interview Surveys) have been used to estimate that about $55.4 \%$ of US adult smokers made a past-year quit attempt ${ }^{1}$. The Centers for Disease Control and Prevention (CDC) have used these data to estimate that the overall prevalence of recent smoking cessation in the US is $7.4 \%$, and that over the past decade rates consistently increased with higher educational attainment, and decreased among the uninsured $(5.2 \%)^{1}$. When considering immediate strategies to achieve the upcoming 2030 goals of adult smoking cessation, a growing body of evidence has identified the workplace as an important site for intervention ${ }^{2,3}$. Participation rates in workplace smoking cessation 
programs are highly variable and range from $12 \%$ to $84 \%$ with a median of $28 \%{ }^{2,3}$. Findings from large scale systematic reviews and meta-regressions, of over 46 studies during the past three decades, indicate that encouraging smoking cessation in the workplace can enhance both participation and even efficacy of longterm smoking cessation ${ }^{4,5}$.

There is, however, wide variation in the type of incentives used and the efficacy of the incentive strategies ${ }^{3,4,6}$. Incentive strategies vary in workplace smoking cessation programming. These strategies include models such as individual financial rewards, group-based lottery rewards, and voluntary 'depositbased' withholding of a pre-specified amount from the payroll ${ }^{3,7}$. These incentives are often used in combination with behavioral smoking cessation interventions offered at the workplace ${ }^{4,5,8-10}$.

In a recent landmark trial, 2538 smokers, identified from the CVS Caremark pharmacy retail employee population, were randomized to one of four types of incentive programs (group vs individual; reward vs deposit-based) or usual care for smoking cessation. Despite randomization, the trial design allowed subjects to accept or reject their assignment. The trial found a very high participation in reward-based smoking cessation programs $(90 \%$ accepting direct cash payment per time point where abstinence from smoking is confirmed at testing) compared to deposit-based incentive programs (13\% accepting a program where they provided a deposit that was refunded upon confirmed abstinence). Overall, reward-based incentive programs were also shown to be the most effective for accomplishing employee smoking cessation in the trial.

In the present study, we examined the participation rate of the Loma Linda University Health (LLUH) BREATHE program - the workplace smoking cessation program offered to all LLUH employees. LLUH is a non-profit, faith-based, academic health system comprising eight health science schools, a medical center with six regional affiliates and a children's hospital, all located in Southern California. Before 2014, LLUH BREATHE was offered to employees who smoked and participation was voluntary and free with no incentive. Participation was estimated at less than $5 \%$.

During 2014-2015, LLUH tested the effect of an innovative, reward-based incentive feature that was added to the LLUH BREATHE program to enhance program participation and efficacy. Specifically, LLUH BREATHE was offered in an incentive manner with lower out-of-pocket costs (lower monthly employee contributions, lower prescription copayments, LLUH BREATHE program at no cost) for the employer-provided health plan. In the present study we focused on the 415 employee smokers who were: 1) identified during a 2014 biometric screening of 13081 benefit eligible LLUH employees and a health claims analysis; and 2) offered participation in LLUH BREATHE through a lower cost health plan option. The objectives of the study are: 1) measure the participation rate and efficacy of LLUH BREATHE program that was provided under a reward-based incentive of lower 'employee out-ofpocket' health plan costs; 2 ) determine demographic and health variables associated with participation in LLUH BREATHE.

\section{METHODS}

\section{Study population}

For the 2014 health plan year, benefit eligible LLUH employees were offered two health plan options: 1 ) The Wholeness Health Plan, and 2) The Base Health Plan. The Wholeness Health Plan had 50-53\% lower out-of-pocket costs (monthly employee contributions, co-payments, co-pay for prescriptions) relative to the Base Plan. To qualify for the Wholeness Health Plan, employees, and covered spouses, needed to complete a health risk assessment (HRA), biometric screening, and complete a care management program, if identified, which included smoking cessation for smokers. In contrast, the Base Health Plan - with the higher out-of-pocket costs - did not require participation activities, and thus employee smokers who chose this plan were not required to participate in smoking cessation.

During biometric screening of 13081 benefiteligible employees, 415 tobacco users were identified through their self-reported health risk assessment data and through claims data. Employee smokers identified through this assessment had the option of appealing against the findings of this assessment by undergoing am exhaled carbon monoxide ( $\mathrm{CO}$ ) test, and those with $\mathrm{CO}$ scores $<10 \mathrm{ppm}$ were re-classified as non-smokers.

This group of 415 employee smokers who were 
offered participation in workplace smoking cessation through a choice of the Wholeness Health Plan (described above) became the analytic sample for the present study and known as the LLUH BREATHE cohort. The secondary analysis study of these health plan data was approved by the Institutional Review Board of Loma Linda University.

\section{Survey, interview and bio-specimen measures} The entire LLUH BREATHE cohort underwent biometric screening that included: 1) anthropometric measures (height, weight, waist circumference, BMI); 2) biomarker collection (blood pressure, triglycerides, HDL, LDL, total cholesterol, fasting and non-fasting glucose); and 3) demographic variables. Participants in the workplace smoking cessation program (by voluntary choice of the Wholeness Health Plan) underwent further baseline survey interviews to ascertain current tobacco use, tobacco dependence (Fagerström index variables), coping strategies, sources of encouragement, and desire and confidence to be tobacco-free. This survey was also administered after the 4-month smoking cessation intervention.

\section{Two smoking cessation interventions}

Cohort members who participated in the intervention were asked to complete two electronic survey forms (Intake/Pre- and Post-intervention), two physician visits, and either an online or in-person group version of the American Lung Association (ALA) 'Freedom from Smoking' course. This was an 8 -week online course that educated participants on the benefits of smoking cessation and assisted individuals in setting a 'quit date'. This program consisted of 8 weekly sessions that took approximately 1 hour to complete. To improve participation and completion rates, multiple messages were sent through mail, email and telephone to each potential participant. Participants were moved to the Base Health Plan if they did not complete their online intake form, missed their 1st physician visit, or missed the final deadline. A survey interview was completed at the end of the program in which a subject's self-report of current smoking status and intensity of tobacco use were compared to the baseline results. This comparison was used as part of assessing efficacy.

\section{Statistical analysis}

Participation rates of the LLUH BREATHE cohort were computed as proportions and confidence intervals were computed using Fleiss's Quadratic method with a continuity correction ${ }^{11}$. Under the intent-to-treat principle, participants were defined as those employee smokers, and/or their covered spouse who smoked, that chose the wholeness health plan at the time it was offered. Those participants who were discontinued from employee smoking cessation due to non-completion of program activities continued to be assessed as 'participants' for the calculations in this report. Among participants, efficacy of the program was determined by computing the proportion and confidence interval reporting no cigarette smoking after 4 months on the program. Intensity of smoking habit (number of cigarettes smoked) was also compared between baseline and 4 months follow-up using a paired t-test.

To examine factors associated with participation in the Wholeness Health Plan in the LLUH BREATHE cohort, we conducted logistic regression analysis with participation as the binary outcome variable. We tested demographic (age, gender, employee/ spouse) and biometric (anthropometric, metabolic blood panel) variables to test hypotheses about gender and 'sick quitter' effects on participation seen in other employee smoker samples. All analyses were conducted using the Statistical Analysis Software $(\mathrm{SAS}){ }^{\circledR}$ version 9.4 .

\section{RESULTS}

\section{Participation rates}

The LLUH BREATHE cohort consisted of 415 employee smokers (aged 24-76 years) who were identified as tobacco users through health risk assessment and medical claims data. Of the 415 smokers, 302 voluntarily chose the smoking cessation through their choice of Wholeness Health Plan and this produced a participation rate of $72.7 \%$ (95\% CI: $69-77 \%)$.

In Table 1, we provide a comparison of demographic and biometric characteristics of participants and nonparticipants. We found that subjects who enrolled in the intervention had a significantly lower blood pressure.

\section{Variables associated with participation}

In Table 2, we present the findings from univariable logistic regression models testing the association 
Table 1. Demographic and biometric characteristics of the Loma Linda University Health BREATHE cohort of employee smokers given by participation/non participation in workplace smoking cessation in 2014

\begin{tabular}{|c|c|c|}
\hline \multirow[t]{2}{*}{ Variables } & $\begin{array}{l}\text { Participants } \\
\qquad(\text { n - 302 })^{a}\end{array}$ & $\begin{array}{c}\text { Von- } \\
\text { participants } \\
(\text { (n-113) }\end{array}$ \\
\hline & $\begin{array}{c}\text { Mean }=\text { SD } \\
\text { or } \%\end{array}$ & $\begin{array}{c}\text { Vean }=\text { SD } \\
\text { or } \%\end{array}$ \\
\hline Age (years) & $46.2 \pm 11.57$ & $47.0 \pm 11.3$ \\
\hline $\begin{array}{l}\text { Employee versus spousal } \\
\text { enrollment }\end{array}$ & 50.7 & 58.4 \\
\hline Female & 40.1 & 35.4 \\
\hline BMI $\left(\mathrm{kg} / \mathrm{m}^{2}\right)$ & $29.9 \pm 6.7$ & $29.8 \pm 7.5$ \\
\hline Waist circumference $(\mathrm{cm})$ & $39.3 \pm 6.4$ & $39.3 \pm 7.5$ \\
\hline $\mathrm{DBP}(\mathrm{mmHg})^{\mathrm{b}}$ & $78.7 \pm 11.5$ & $81.2 \pm 11.5$ \\
\hline $\mathrm{SBP}(\mathrm{mmHg})^{\mathrm{b}}$ & $130.0 \pm 18.7$ & $134.5 \pm 18.6$ \\
\hline Total cholesterol (mg/dL) & $180.8 \pm 36.4$ & $182.8 \pm 34.6$ \\
\hline HDL (mg/dL) & $43.9 \pm 14.8$ & $45.8 \pm 16.3$ \\
\hline
\end{tabular}

BMI: body mass index. DBP: diastolic blood pressure. SBP: systolic blood pressure. a Employees and/or their spouses enrolled in a health plan that required participation in workplace smoking cessation. $b \quad p<0.05$ in comparisons using Student's t-test or chi-squared test.

Table 2. Logistic regression models relating demographic and biometric variables to a binary outcome for participation in the Wholeness Health Plan Smoking Cessation Program by LLUH BREATHE cohort members who were current smokers $(\mathrm{N}=\mathbf{4 1 5})$

$\begin{array}{lccc}\text { Variables } & \text { OR } & 95^{\circ} \text { CI } & p \\ \text { Gender } & 0.82 & 0.52-1.28 & 0.38 \\ \text { Age (years) } & 0.99 & 0.98-1.01 & 0.53 \\ \text { Employee versus Spouse } & 0.73 & 0.47-1.13 & 0.16 \\ \text { BMI }\left(\mathrm{kg} / \mathrm{m}^{2}\right) & 1.00 & 0.97-1.03 & 0.88 \\ \text { Waist circumference }(\mathrm{cm}) & 1.00 & 0.97-1.03 & 0.97 \\ \text { DBP }(\mathrm{mmHg}) & 0.98 & 0.96-0.99 & 0.049 \\ \text { SBP }(\mathrm{mmHg}) & 0.99 & 0.98-0.99 & 0.032 \\ \text { Total cholesterol }(\mathrm{mg} / \mathrm{dL}) & 1.00 & 0.99-1.00 & 0.61 \\ \text { HDL }(\mathrm{mg} / \mathrm{dL}) & 0.99 & 0.98-1.01 & 0.26\end{array}$

BMI: body mass index. DBP: diastolic blood pressure. SBP: systolic blood pressure.

between demographic variables, biometric variables and a binary outcome for participation (in smoking cessation through a choice of the Wholeness Health Plan). We found that subjects with lower blood pressure were significantly more likely to participate in the workplace smoking cessation. Males and employees (vs spouses) were less likely to participate in the workplace smoking cessation; this did not, however, attain significance.
Table 3. Multivariable logistic regression testing interaction between spousal and gender contrasts on participation in the Wholeness Health Plan Smoking Cessation Program by LLUH BREATHE cohort members who were current smokers $(\mathrm{N}=415)$

\begin{tabular}{lcccl} 
Variables & $\begin{array}{c}\text { Participation } \\
(\%)\end{array}$ & OR $^{*}$ & $95^{\circ}$. CI & \multicolumn{1}{c}{ D } \\
Male employee & 55.7 & 1.00 & Ref. & \\
Male spouse & 77.2 & 2.89 & $1.59-5.24$ & 0.0005 \\
Female employee & 76.5 & 2.71 & $1.47-5.00$ & 0.001 \\
Female spouse & 58.3 & 1.14 & $0.33-3.95$ & 0.84
\end{tabular}

*Model age adjusted

We also built an age-adjusted multivariable model to examine gender and spousal contrasts (findings not shown). Here, we found that both male smokers (compared to females) and employee smokers (relative to spouse smokers) were significantly less likely to participate. We further investigated this by testing an interaction of gender and spousal contrasts in Table 3. In this model, we found that the male employees who smoked were least likely to participate and were used as the reference group for the model. We found that smokers who were female employees, or the male spouses of female employees, were two-fold more likely to participate in the program relative to male employee households.

\section{Self-reported point prevalence of smoking cessation at 4 months}

Although efficacy of the intervention is best assessed in a randomized controlled trial, we did, on an exploratory uncontrolled basis, assess the efficacy of the smoking cessation intervention in participants using the self-report of cigarette smoking on the follow-up questionnaire administered after the 4-month intervention. Of the 302 participants, the analytic sample for efficacy assessment reduced to 263 participants due to subjects who were discontinued on the health plan were no longer employed by the organization, or opted out of the requirements and chose the Base Health Plan. Under a 'per protocol' principle of focusing on those subjects that remained on the Wholeness Health Plan protocol and employed at LLUH $(n=263)$, the point prevalence of smoking cessation at 4 months was 55\% (95\% CI: $49-61 \%$ ). Under an intention-to-treat principle $(n=302)$ of 
including all subjects who participated, the point prevalence of smoking cessation at 4 months among these subjects was $48 \%$ (95\% CI: $42-54 \%$ ).

\section{DISCUSSION}

Our findings from 415 current smokers in the LLUH BREATHE cohort indicate a very high rate of participation (73\%) in employer-sponsored smoking cessation compared to the $28 \%$ median participation rate computed across 23 studies of employee smoking cessation programs in affluent nations ${ }^{3}$. One potential reason for the uniquely high participation rate in our study is that participation in workplace smoking cessation program was encouraged through a rewardbased method of lowering employee 'out-of-pocket' costs for the employee; a health plan reduction (50-53\%) in monthly premiums and co-pays, such as outpatient care and prescriptions, and no out-ofpocket costs for the workplace smoking cessation program or nicotine replacement therapy. The very high rate of participation did, however, vary by gender and spousal effects whereby male employee households with a qualifying smoker (employee or spouse) were least likely to participate in smoking cessation. For the LLUH BREATHE smokers who participated, the point prevalence smoking cessation rate at 4 months was $48 \%$ using group or online versions of the American Lung Association Freedom from Smoking program.

\section{Encouraging successful smoking cessation}

One factor contributing to the high participation rate in the LLUH BREATHE cohort in smoking cessation was the use of an individual 'reward-based' incentive (as opposed to 'deposit-based' incentive) - a method that has been shown in two large systematic reviews ${ }^{3,5}$ and one large randomized controlled trial ${ }^{6,7}$ to be very effective in accomplishing both participation and higher rates of smoking cessation ${ }^{10,12}$.

LLUH BREATHE participants promoted smoking cessation by enrolling in the health plan that contained reduced monthly out-of-pocket contribution, prescription drug costs and co-payments - thus tangibly increasing their individual monthly income. This is a sizable reward amount even when compared to other successful reward-based programs described in the literature. Specifically, in recent analyses of reward-based incentives in workplace smoking cessation programs, individual based rewards were 'one-time' payments ranging from $\$ 10$ up to $\$ 750$ for extended abstinence. Groupbased 'employee lottery' rewards ranged from $\$ 40$ to $\$ 500$, and were only given to one of the abstinent participants.

For the LLUH BREATHE cohort findings, we note that the individual rewards of a long-term reduction in 'out-of-pocket' costs of health coverage (monthly premiums, co-pays) in our study would vary per subject (based on family members covered, number of co-pays for visits/prescriptions) but would likely far exceed the individual rewards reported to date in the literature (i.e. up to $\$ 750$ in some studies). Using the average LLUH health plan cost data we estimate that the cost savings incentive could range on average from $\$ 600$ to $\$ 1200$ per year. Further prospective follow-up of the BREATHE cohort for health plan costs is needed to quantify the effect of this incentive. Moreover, the LLUH BREATHE approach needs discussion relative to proposed changes to national health coverage policies. The federal government has supported US Public Health efforts by aiding nicotine cessation strategies, which can be seen in regulations released in June 2013. These regulations, which were consistent with the Affordable Care Act regarding nondiscriminatory wellness programs in group health coverage, increased the maximum permissible reward from $20 \%$ to $30 \%$ of the cost of health coverage for individuals under a health-contingent wellness program. These regulations also increased the maximum permissible reward to $50 \%$ for wellness programs designed specifically to prevent or reduce tobacco use. This type of increase in the reward, of allowed wellness programs connected to group health plans, would potentially benefit, charge or withhold thousands of dollars a year from health plan members who used tobacco. The LLUH BREATHE approach for workplace smoking cessation in 2014 required participation only (if the Wholeness Health Plan was chosen) and the cost difference of the Base Health Plan was much less than $50 \%$ of the cost of coverage.

Leeks et al. ${ }^{3}$ have proposed a framework for how incentives can enhance participation and efficacy of workplace smoking cessation. The framework divides incentives into two domains: incentive 
participation and incentive long-term abstinence. We note that our method in the LLUH BREATHE cohort employs both domains since choosing the Wholeness Health Plan, as a long-term option, offers the immediate financial incentive to participate and the long-term incentive to remain abstinent in order to avoid having to participate in the program again as required by the health plan. During each open enrollment period for the plan, a current smoker (whether in relapse from the previous program or a non-participant) is again encouraged to choose participation and abstinence through the substantial cost-savings in the wholeness health plan.

\section{Participant demographic and health factors}

Our findings in Table 2 indicating that male employees were significantly less likely to participate confirm the findings of several large meta-analyses of workplace preventive programs and workplace smoking cessation programs ${ }^{3-6}$. In a meta-regression of 23 worksite prevention programs, Robroek et al. ${ }^{5}$ found that women had significantly higher participation than men $(\mathrm{OR}=1.67$; 95\% CI: $1.25-2.27)$, but that this advantage was not apparent for preventive programs on physical fitness (i.e. access to an employersponsored fitness center). Also evident from the metaregression was that participation was in higher multicomponent workplace preventive programs (i.e. diet, smoking, and physical activity programs $)^{5}$. Recently, Halpern et al. ${ }^{6}$ identified, in asecondary analysis of the large CVS Caremark trial, that men were less likely to participate in reward-based workplace smoking cessation programs. Taken together, the findings from our work and other studies highlight the need to find additional programmatic design features to engage and/or encourage male employees in workplace smoking cessation programs. Potentially, including smoking as a multi-component intervention (i.e. adding a physical fitness component) for all participants could be tested as a method to improve participation in men. Mixed-method approaches to identify barriers to participation in men (i.e. privacy concerns, employer intrusion on health) may also be needed ${ }^{8,9}$. Halpern et al. $^{6}$ raise the possibility that deposit-based incentives perform better for workplace smoking cessation programs for men.

Our study did not find that LLUH BREATHE cohort members, who were participants in workplace smoking cessation, had biometric values that indicated poorer health relative to non-participants. Thus, at least based on the biometrics measured, we did not find evidence of a 'sick quitter' effect shown in other studies ${ }^{13}$. If confirmed, this further supports the cost-savings to employers for investing in workplace smoking cessation.

\section{Spousal influences on employee-based smoking cessation}

Our finding of an interaction between spousal contrasts and gender contrasts on participation in workplace smoking cessation is a new finding that needs testing and/or confirmation from other studies. Specifically, we found a much higher participation by the female employees whose male spouse smoked $(\mathrm{OR}=2.89$; 95\% CI: 1.59-5.24), compared with male employees whose female spouse smoked $(\mathrm{OR}=1.14$; 95\% CI: 0.33-3.95). This gender-based heterogeneity in spousal influence needs further investigation.

Overall, studies on health behavior change patterns in couples have shown that both men and women are more likely to make a positive health behavior change, if the partner is also engaged in health behavior change ${ }^{14}$, particularly in the area of smoking ${ }^{15}$. The Health and Retirement Study concluded that partnership in smoking cessation influences not only the time of quitting but also a long-term adherence to a healthier behavior ${ }^{16}$.

\section{Limitations}

A number of limitations of this study need consideration. We have assessed the point prevalence of smoking cessation at 4 months using selfreported cessation data. In a random sample of 100 participants, we did, however, find a cessation rate that exceeded $40 \%$, which continues to exceed US norms for smoking cessation rates in adults. We note that efficacy is not measured by a randomized controlled trial design but rather by smokers who chose to participate in a smoking cessation program that is encouraged through the health plan choice. However, we note the ethical issues inherent in a randomized design that would deny smokers in the organization a wholly subsidized model for smoking cessation that has proven to be effective. Thus, our observational approach may in fact be the only design that can ethically test participation rates in a smoking 
cessation program that has a novel incentive that is built into health plan coverage. Lastly, we note that LLUH employees represent a sample of employed adults from an organization consisting of eight health science schools, six hospitals, and a physician practice corporation. The high prevalence of adults with higher education contributes to: 1) a lower prevalence of smoking, and 2) the need to investigate the effect of health plan incentives in employee cohorts with a wider range of sociodemographic characteristics.

\section{CONCLUSIONS}

Our findings identify that workplace smoking cessation that uses a novel, reward-based incentive of lower 'out-of-pocket' health plan costs produced a participation rate that was much higher than US norms. Despite the overall high participation, our study identified that further work is needed to design programs that specifically engage male employees and employee spouses in workplace initiatives to promote smoking cessation.

\section{REFERENCES}

1. Babb S, Malarcher A, Schauer G, Asman K, Jamal A. Quitting Smoking in Adults - United States, 2000-2015 MMWR Morb Mortal Wkly Rep. 2017;65(52):1457-1464. doi:10.15585/mmwr.mm6552a1

2. van den Brand FA, Magnée T, de Haan-Bouma L, Barendregt C, Chavannes NH, vanSchayck OCP, Nagelhout GE. Implementation of Financial Incentives for Successful Smoking Cessation in Real-Life Company Settings: A Qualitative Needs Assessment among Employers. Int J Environ Res Public Health. 2019;16(24):E5135. doi:10.3390/ijerph16245135

3. Leeks KD, Hopkins DP, Soler RE, et al. Worksitebased incentives and competitions to reduce tobacco use. A systematic review. Am J Prev Med. 2010;38(2 Suppl):S263-S274. doi:10.1016/j.amepre.2009.10.034

4. Cahill K, Hartmann-Boyce J, Perera R. Incentives for smoking cessation. Cochrane Database Syst Rev. 2015(5):Cd004307. doi:10.1002/14651858.CD004307.pub5

5. Robroek SJ, van Lenthe FJ, van Empelen P, et al. Determinants of participation in worksite health promotion programmes: a systematic review. Int J Behav Nutr Phys Act. 2009;6(1):26. doi:10.1186/1479-5868-6-26

6. Halpern SD, French B, Small DS, et al. Heterogeneity in the Effects of Reward- and Deposit-based Financial Incentives on Smoking Cessation. Am J Respir Crit Care Med. 2016;194(8):981-988. doi:10.1164/rccm.201601-01080C

7. Halpern SD, French B, Small DS, et al. Randomized trial of four financial-incentive programs for smoking cessation. N Engl J Med. 2015;372(22):2108-2117.
doi:10.1056/NEJMoa1414293

8. Rongen A, Robroek SJ, van Ginkel W, et al. Barriers and facilitators for participation in health promotion programs among employees: a six-month follow-up study. BMC Public Health. 2014;14(1):573. doi:10.1186/1471-2458-14-573

9. Rongen A, Robroek SJ, van Lenthe FJ, et al. Workplace health promotion: a meta-analysis of effectiveness. Am J Prev Med. 2013;44(4):406-415. doi:10.1016/j.amepre.2012.12.007

10. Mache S, Vitzthum K, Groneberg DA, Harth V. Effects of a multi-behavioral health promotion program at worksite on smoking patterns and quit behavior. Work. 2019;62(4):543-551. doi:10.3233/WOR-192889

11. Fleiss JL. Statistical Methods for Rates and Proportions. 2nd ed. New York, NY: John Wiley \& Sons; 1981. doi:10.1002/bimj.19740160814

12. Notley C, Gentry S, Livingstone-Banks J, Bauld L, Perera $\mathrm{R}$, Hartmann-Boyce J. Incentives for smoking cessation. Cochrane Database Syst Rev. 2019;7:CD004307. doi:10.1002/14651858.CD004307.pub6

13. Stolz D, Scherr A, Seiffert B, et al. Predictors of success for smoking cessation at the workplace: a longitudinal study. Respiration. 2014;87(1):18-25. doi:10.1159/000346646

14. Jackson SE, Steptoe A, Wardle J. The influence of partner's behavior on health behavior change: the English Longitudinal Study of Ageing. JAMA Intern Med. 2015;175(3):385-392. doi:10.1001/jamainternmed.2014.7554

15. Cobb LK, McAdams-DeMarco MA, Huxley RR, et al. The association of spousal smoking status with the ability to quit smoking: the Atherosclerosis Risk in Communities Study. Am J Epidemiol. 2014;179(10):1182-1187. doi:10.1093/aje/kwu041

16. Margolis R, Wright L. Better Off Alone Than With a Smoker: The Influence of Partner's Smoking Behavior in Later Life. J Gerontol B Psychol Sci Soc Sci. 2016;71(4):687-697. doi:10.1093/geronb/gbu220

\section{CONFLICTS OF INTEREST}

The authors have completed and submitted the ICMJE Form for Disclosure of Potential Conflicts of Interest and none was reported.

\section{FUNDING}

The study was funded by a Loma Linda University Risk Management Health Plan award given to the Center for Health Research at the School of Public Health at Loma Linda University.

PROVENANCE AND PEER REVIEW

Not commissioned; externally peer reviewed. 